\title{
CONGENITAL RIGHT-SIDED DIAPHRAGMATIC HERNIA
}

\section{SOME DIFFICULTIES IN DIFFERENTIAL DIAGNOSIS AND OPERATIVE REPAIR}

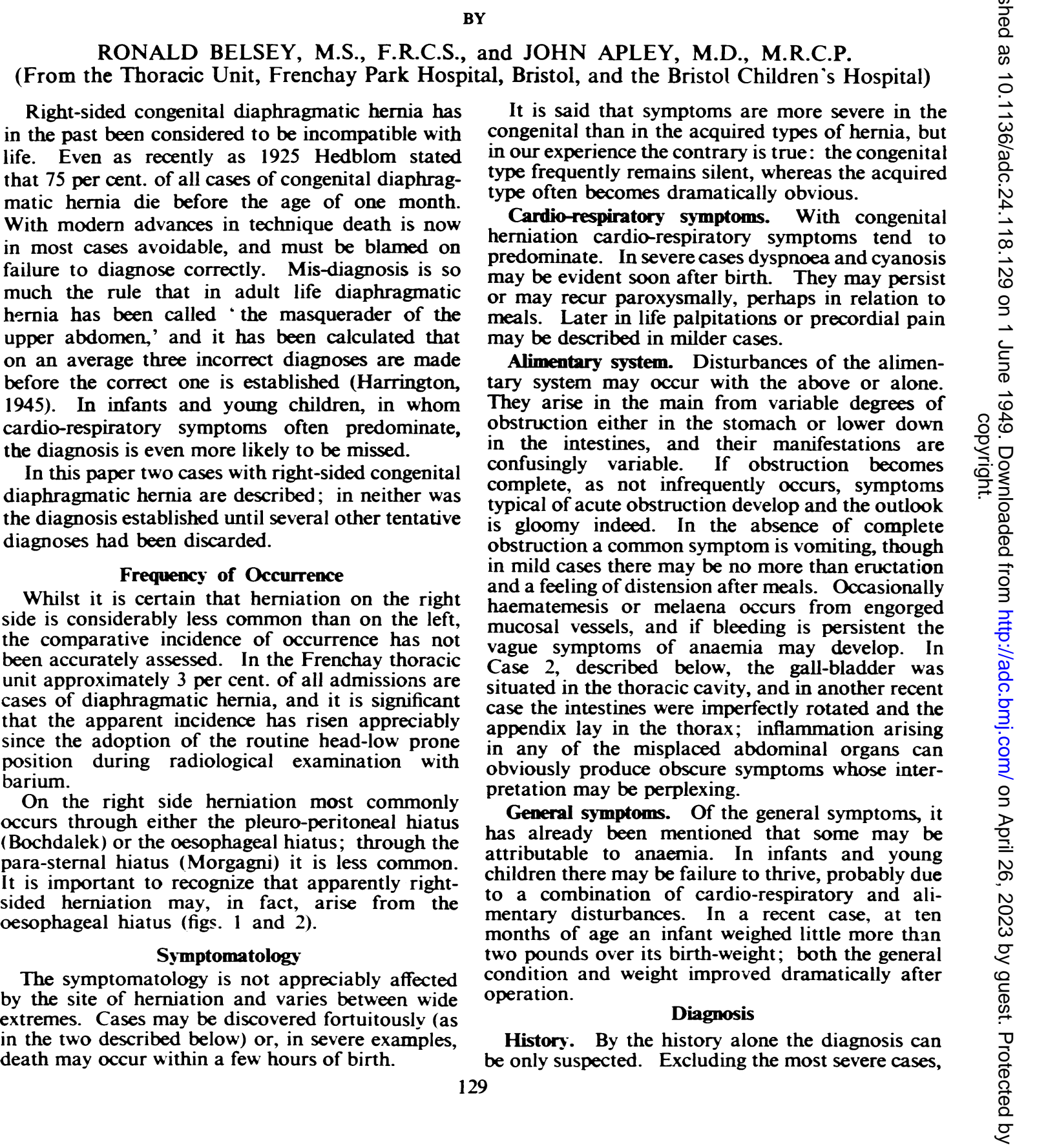


the common story (as in Case 2) is that of so-called recurrent pneumonia. This erroneous diagnosis is usually based on the evidence of dyspnoea and abnormal physical signs obtained when the child is examined because of some unrelated upper respiratory infection. Apparently inexplicable vomiting which recurs over a considerable pericd should also suggest the diagnosis.

Clinical examination. This may, on occasion, provide an unequivocal diagnosis. The essential feature is the detection of bowel sounds in the chest. To elicit this pathognomonic sign it is advisable to auscultate soon after the patient has taken a meal, and to continue auscultation for at least ten minutes. In both the cases described with these precautions the characteristic bowel sounds were invariably heard; without them they were frequently not detected. It has long been realized that even in the presence of a known diaphragmatic hernia abnormal signs in the chest may be absent; possibly the observance of the above precautions would reduce the number of such failures.

In addition, the variability of the physical signs from time to time in the same patient is itself suggestive of the diagnosis. Impairment of expansion on the affected side, dullness to percussion, and suppression of breath sounds, constitute the usual findings in the chest, and the mediastinum may be displaced. Occasionally the signs simulate those of pneumothorax or hydro-pneumothorax. The liver may lie in the thoracic cavity, and the physical signs which it produces are very commonly attributed to pleural effusion. If the duodenum is obstructed and the stomach consequently dilated, a characteristic splash may be elicited. When large portions of the bowel are contained in the chest the abdomen may fail to develop proportionately and may present a scaphoid appearance.

\section{Radiological Appearances}

In every suspected case radiological studies are essential, especially in view of the danger inherent in diagnostic aspiration where portions of the bowel are contained in the chest.

Only conditions occurring on the right side which are commonly confused will be considered here, though in theory similar appearances might be produced by 'gas' infections of the pleura, eventration of the right diaphragm with upward protrusion of part of the liver, multiple tuberculous cavities, congenital cystic disease of the lungs, hydatid cysts that have ruptured into the bronchial tree, and diverticula of the oesophagus.

The radiological appearances are suggestive because of their very oddity. There may be shadowing at the base which is "typical of nothing. The consistency of the shadow is not completely uniform, with a rare exception: if omentum alone has herniated the opacity may appear homogeneous. An appearance of 'tenting' is not unusual (fig. 3). If lateral views of the chest are studied in conjunction with antero-posterior views there should be little difficulty in distinguishing herniation from free pleural effusion.

The commonly accepted statement that radiological diagnosis can be made easily by three features, the detection of gas bubbles, collapsed lung, and mediastinal displacement, misleads by over-simplification.

In most cases diagnosis is indeed suggested by the presence of gas shadows and the characteristic pattern caused by the valvulae conniventes of the small intestines and the haustration of the large but this appearance may be simulated by (1) lung abscesses or saccular bronchiectasis (figs. 4 and 5); (2) loculated or encapsulated effusions; (3) loculated sub-diaphragmatic abscess; (4) interposition of colon between liver and diaphragm (fig. 6) or, (5) congenital abnormalities of the oesophagus. Collapse of lung and mediastinal displacement occur in varying regree, but are only subsidiary in diagnosis.

Certainty of diagnosis can be achieved by means of barium follow-through studies (figs. 7, 8, 12, 13). In no case should the precaution be omitted of tilting the patient in the prone position with the head low during the examination.

Lateral views with barium in the intestine help in localizing the position of the diaphragmatic defect, but to assess the size of the defect from radiological appearances is usually impossible.

\section{Treatment}

The treatment of the condition is always surgical. It should be undertaken as early as possible to avoid first, the technical difficulties caused by failure of expansion of the abdomen, which becomes progressively and relatively more marked the longer the abdominal contents remain in the thorax; second, the general maldevelopment which accompanies long standing disturbances of intestinal function; and third, the occurrence of acute intestinal obstruction. If obstruction does occur, surgical treatment becomes a matter of extreme urgency.

Two routes are available, the abdominal and the thoracic. The thoracic approach permits a sounder repair of large diaphragmatic defects, and an increase in the size of the abdominal cavity by raising the attachments of the diaphragm to a higher level in the thorax. Following replacement of the abdominal viscera the lung can be expanded without difficulty by the anaesthetist, and any structural change in the lung following the prolonged compression by displaced abdominal viscera is unusual.

In both thecases reported the defect was so large that the usual repair with an overlap was out of the question. Various methods of overcoming such a difficulty have been devised. Harrington's (1948) technique of performing a basal thoracoplasty to free the diaphragm has the disadvantage of being a deforming operation which interferes permanently with the function of the affected side of the chest. A new procedure was evolved, and proved successful in both cases. The chest was entered through the bed 
of the ninth rib. After replacement of the abdominal viscera, the remnants of the diaphragm were freed from their attachments to the costal margin all round, and left with only their mediastinal origin and corresponding blood and nerve supply intact. The diaphragmatic defect was then closed by two rows of interrupted sutures of stainless steel wire and an overlap. The repaired diaphragm, much reduced in size, was reattached to the thoracic wall at a level two interspaces higher than normal with interrupted sutures of the same material, no difficulty being encountered in bridging the lesser distance horizontally across the thoracic cavity, after eliminating the costo-phrenic sulcus. The phrenic nerve was not crushed in either case, and the functional result has proved excellent in both patients.

\section{Case Reports}

Case 1. A girl aged 7 years was found on routine chest examination by a school medical officer to have abnormal physical signs in the right chest. She was sent to the local tuberculosis dispensary where a radiograph of the chest was taken. A diagnosis of right-sided pleural effusion was made, and the child was admitted as an emergency to a children's sanatorium with instructions to the resident medical officer to ' aspirate the chest.' The resident medical officer elicited from the mother a history of recurring bouts of abdominal pain and vomiting. The attacks had been irregular and bore little relationship to meals. There were no symptoms suggestive of pulmonary disease. Examination revealed an under-nourished and under-developed child; the lower half of the right chest was dull to percussion and breath sounds were replaced by bowel sounds, but this sign was elicited only on prolonged auscultation. The abdomen was scaphoid in appearance. A radiograph revealed a dense shadow in the lower half of the right chest with a few gas-containing areas showing typical intestinal haustration towards its peaked upper margin (fig. 3). A barium followthrough examination revealed the presence of half the duodenum, which was partially obstructed, the whole of the small intestine, and the right half of the colon within the right pleural cavity. Had thoracentesis been performed before an accurate diagnosis had been made, disaster would almost surely have followed.

The child was transferred to the Frenchay thoracic unit and, after a preliminary course of breathing exercises lasting four weeks, the hernia was repaired through the thoracic approach. No hernial sac was present. Replacement of the viscera was difficult owing to the contraction of the abdomen. The defect in the diaphragm, a patent pleuroperitoneal canal, was so large that the plastic repair with transposition to a higher level, as already described, was necessary. The lung was inflated without difficulty and the chest closed without drainage. Convalescence was uneventful. The child has remained free from symptoms, and examination five years later showed normal development. $\mathrm{X}$-ray examination shows that the right diaphragm moves normally, the right lung is fully expanded, and there is no impairment of thoracic function (fig. 9).

Case 2. A girl aged 4 years was admitted to hospital with a diagnosis of right basal 'pneumonia.' The mother reported two similar attacks of pneumonia, also on the right side, during the previous year. On admission the child was pale, looked ill, and had an intermittent cough; the temperature was $101^{\circ} \mathrm{F}$., and the pulse rate 130 , and respirations 40 per minute. Examination revealed poor movement, impaired percussion note, diminished breath sounds, and poor vocal fremitus at the right base. A diagnosis of pneumonic consolidation with a pleural effusion was made. Penicillin therapy was commenced. Eleven days after admission the dullness at the right base was less marked and bowel sounds in the chest were detected for the first time. Review of the radiographs suggested the presence of intestines, containing mostly fluid but also a little gas, within the right pleural cavity (figs. 10 and 11).

Further x-ray examination showed appearances more typical of a diaphragmatic hernia, and a barium follow-through revealed that the whole of the small bowel and the right half of the colon were in the right pleural cavity (figs. 12 and 13). The temperature, pulse rate, and respiration rate quickly dropped to normal, and penicillin was discontinued after twelve days. Breathing exercises were started.

Four weeks after admission a right thoracotomy was performed through the bed of the ninth rib. No hernial sac was present. Part of the duodenum, the whole of the small bowel, the right half of the colon, and the liver lay free in the pleural cavity. The right lung was collapsed. The gall bladder was in contact with the axillary part of the thoracic wall. Any attempt to needle the chest might have produced bizarre and dramatic consequences. Following replacement of the abdominal viscera the defect was again found to be too large for a simple repair, and a similar operation to that performed in the previous case was necessary. Convalescence was uneventful. The child has remained free from symptoms since the operation, her general condition is excellent, and on x-ray and screening the recon. structed right diaphragm appears to function normally (figs. 14 and 15).

\section{Summary}

1. The symptomatology, diagnosis, and differential diagnosis of right-sided congenital diaphragmatic hernia are reviewed.

2. A new technique for the plastic repair of large diaphragmatic defects is described.

3. Two cases successfully treated surgically are reported.

\section{REFERENCES}

Hedblom, C. A. (1925). Jour. Amer. med. Ass., 85, 947. Harrington, S. W. (1945). Ann. Surg., 122, 546. (1948). Surg. Gynec. Obstet.. 86, 735. 


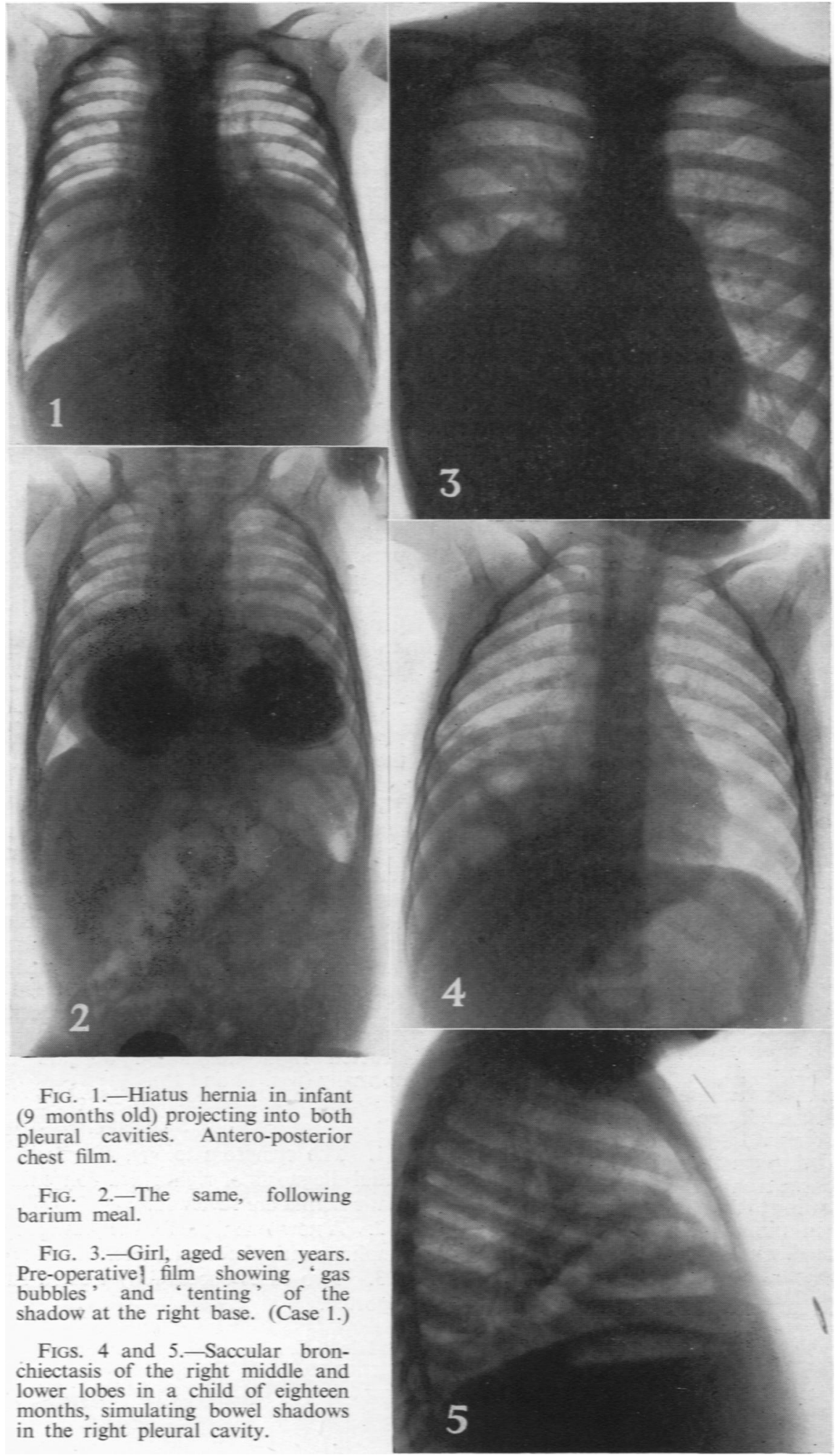


FIG. 6.-Interposition of colon between the liver and

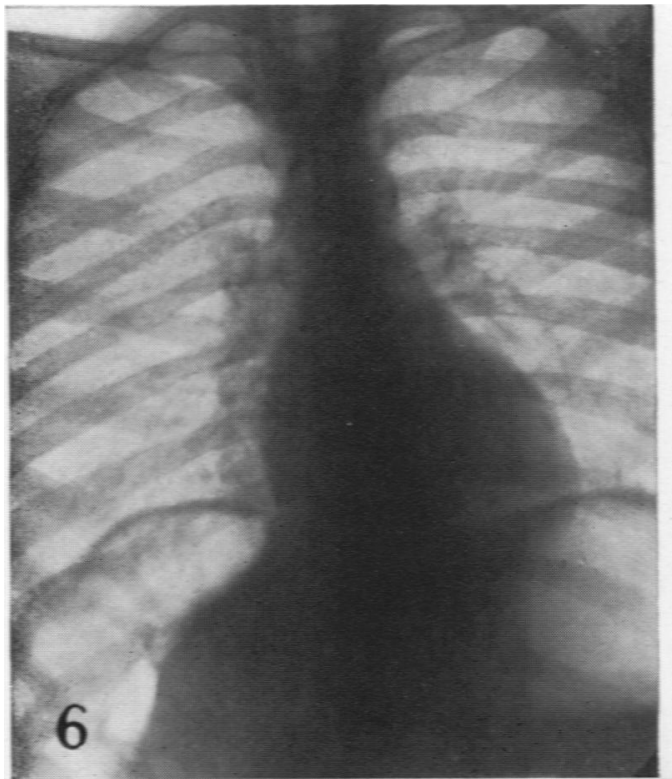

Fig. 6.-Interposi
the right diaphragm.

FIGs. 7 and 8.-Barium meal showing small intestine lying freely in the right pleural cavity.

FIG. 9.-Antero-posterior chest film five years after operation. (Case 1. Five years after operation.)
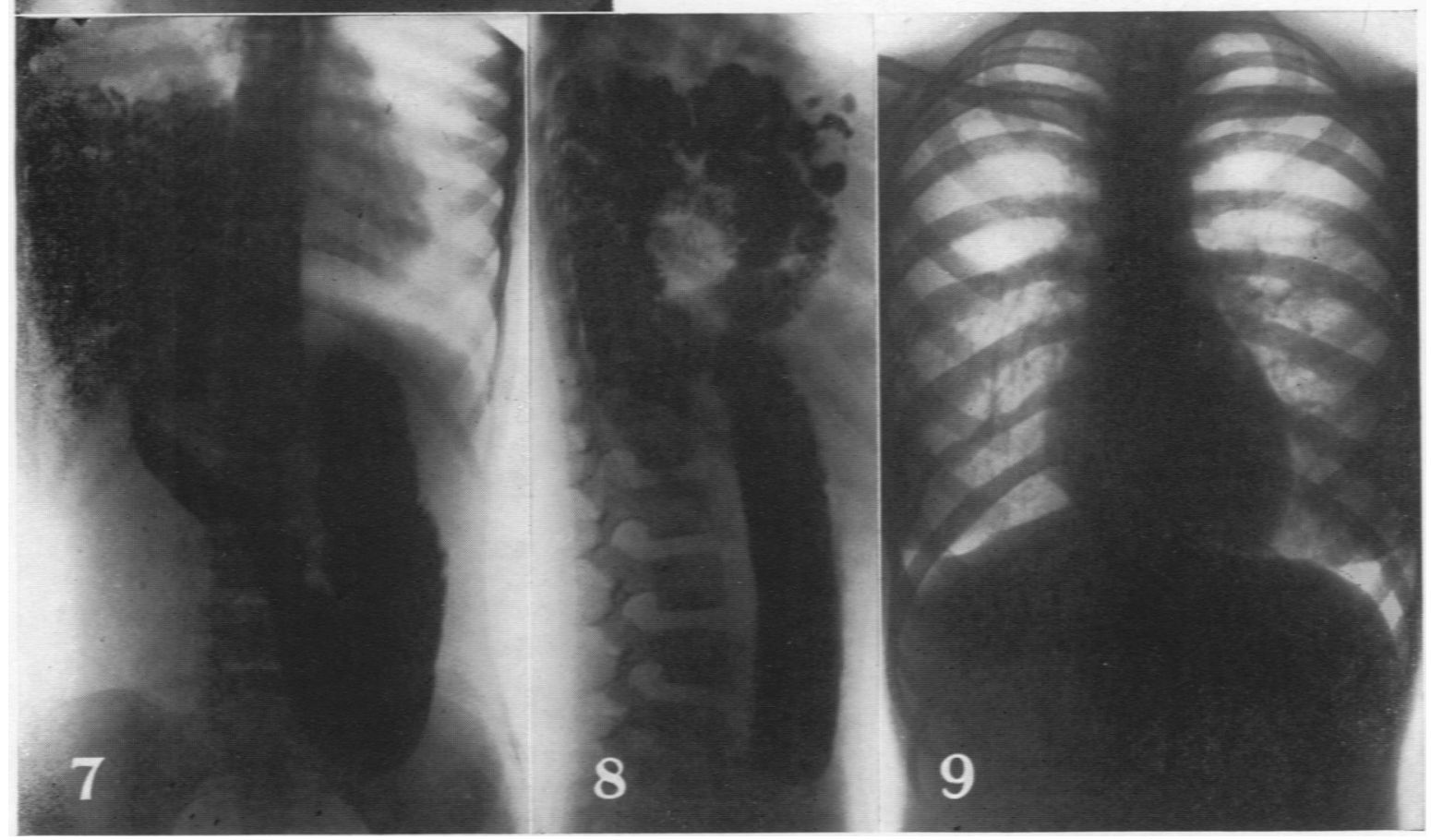


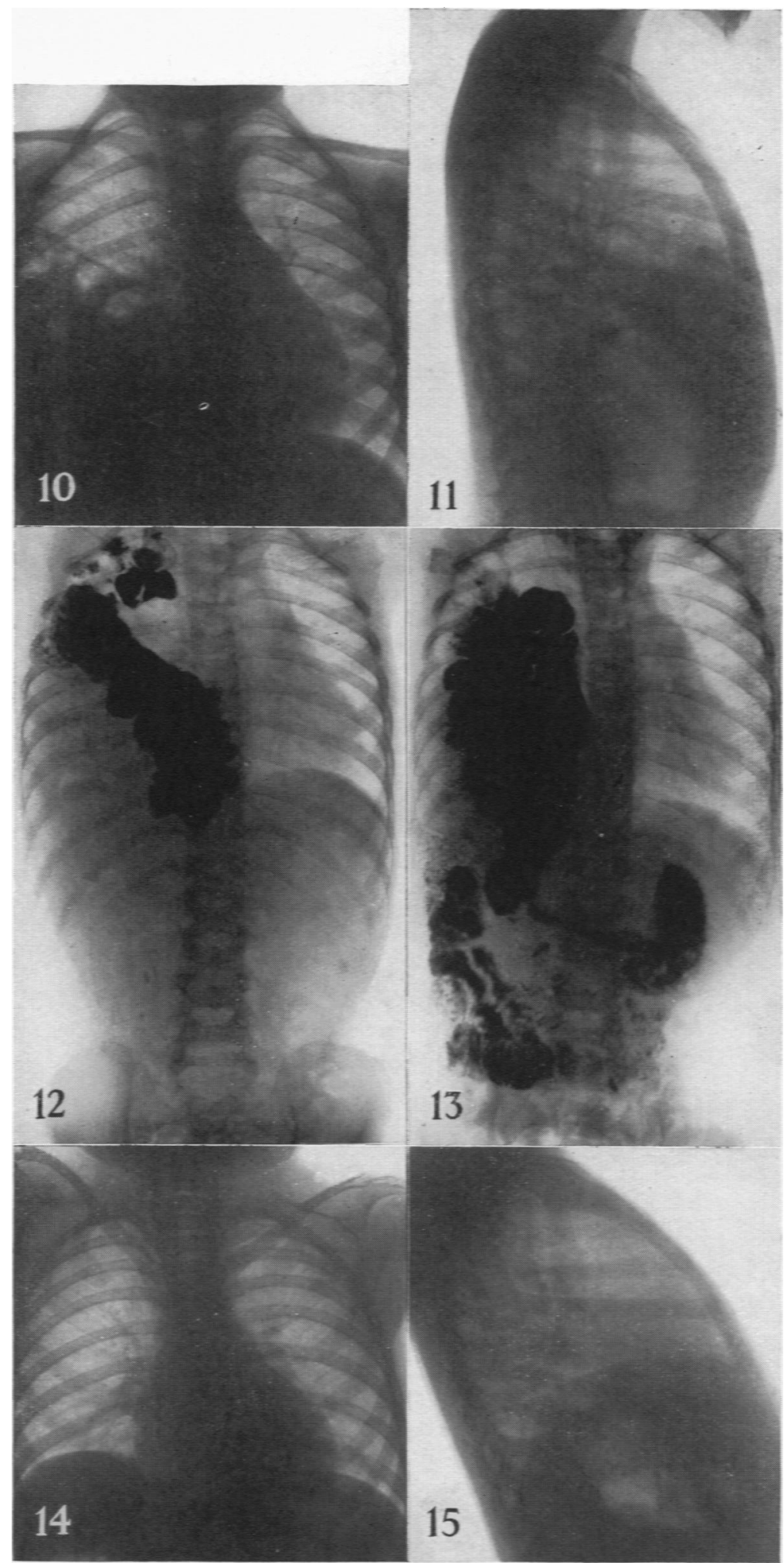

Figs. 10 and 11.-Case 2.) Antero-posterior and lateral films of chest showing 'gas bubbles' and 'tenting' of the opacity at the right base.

Figs. 12 and 13.-Case 2.) Barium meal in prone head-low position; both small and large intestine lying free in right pleural cavity.

Figs. 14 and 15.- (Case 2.) Post-operative film of chest following repair; right diaphragm functioning normally. 